\title{
Evaluación del estado de salud con la Encuesta SF-36: resultados preliminares en México*
}

Miguel A.Zúniga, M.D., D r. P.H.,1) Genny T. C arrillo-Jiménez, M.D., Sc.D., ${ }^{(2)}$ Peter J. Fos, D.D.S., Ph. D., ${ }^{(1)}$

Barbara Gandek, M.S., ${ }^{(3)}$ Manuel R. Medina-Moreno, M.C. ${ }^{(4)}$

Zúniga MA, Carrillo-Jiménez GT, Fos PJ, Gandek B, Medina-Moreno MR. Evaluación del estado de salud con la Encuesta SF-36: resultados preliminares en México. Salud Publica Mex 1999;41:110-118.

\section{Resumen}

Objetivo. Establecer, con la Encuesta SF-36, un perfil multidimensional del estado de salud de una población del sureste de México y analizar las propiedades psicométricas de una traducción de la Encuesta SF-36 autorizada por el Proyecto Internacional de Evaluación de la Calidad deVida. Material y métodos. La SF-36 se aplicó a 257 participantes voluntarios de una clínica médica y a un grupo control de una institución pública gubernamental. Se utilizó, sin modificar, la metodo logía de construcción de las escalas de salud propuestas por los autores de la encuesta. Se analizó psicométricamente la validez y la confiabilidad de la adaptación de la SF-36 para su uso en México. Resultados. Se construyeron ocho escalas o conceptos de salud relacionados con función física, rol físico, dolor corporal, salud general, vitalidad, función social, rol emocional y salud mental. En los participantes de los servicios médicos la escala con más bajo promedio fue la de salud general (63), y la más alta, la de rol físico (89). En la población control la escala con promedio más alto fue función física (94.6), y la más baja, salud general (73). La comparación de promedios de escalas en ambos

\author{
Zúniga MA, Carrillo-Jiménez GT, Fos PJ, \\ Gandek B, Medina-Moreno MR. \\ Health status evaluation \\ with the SF-36 Survey: \\ Preliminary results in Mexico. \\ Salud Publica Mex 1999;41:110-118.
}

\section{A bstract}

Objective. To establish a multidimensional profile of the health status in a population of southeastern Mexico and analyze the psychometric properties of a translation of the SF-36 survey authorized by the International Q uality of Life Assessment Project. Material and methods. The SF-36 was administered to 257 volunteers of a clinic and a control group in a governmental institution. The methodology proposed by the author of the survey to build the health scales was used without modifications. The validity and reliability of the SF-36 adaptation to Mexico was psychometrically analyzed. Results. Eight scales or health concepts related to physical function, physical role, body pain, general health, vitality, social function, emotional role and mental health were constructed. In the health service volunteers, the scale with the lowest mean was general health (63), and that with the highest (89) was physical role. In the control population, the lowest mean was general health (73) and the highest (94.6) was physical function. A comparison of the health scales of both groups showed significant differences in physical function, physical role, body pain, general

Los resultados de este estudio fueron presentados bajo el título: "Establecimiento de N ormas Poblacionales del Estado de Salud en México: uso de la Encuesta de Salud SF-36", en International Society ofTechnology A ssessment in Health C are,Twelfth A nnual Meeting, San Francisco, C alifornia, junio 23-26, 1996.

Este trabajo se llevó a cabo con el apoyo de las siguientes instituciones:The D epartment of Health Systems Management deTulane University Medical Center, el C entro de Investigaciones Regionales D r. Hideyo N oguchi de la Universidad Autónoma de Yucatán y The Health Institute del N ew England Medical Center.

(1) Departamento de Gerencia de Sistemas de Salud, Tulane University School of Public Health and Tropical Medicine, N ueva O rleans, LO, Estados Unidos de América (EUA).

(2) Centro de Investigaciones Regionales, Dr. Hideyo N oguchi, Universidad Autónoma de Yucatán, México.

(3) International Q uality of Life Assessment Project, Boston, Mass., EUA.

(4) Facultad de Medicina, Universidad Autónoma de Yucatán, México. 
grupos de participantes mostró diferencias estadísticamente significativas en función física, rol físico, dolor corporal, salud general y vitalidad. Conclusiones $C$ on base en los resultados de la evaluación psicométrica, la SF-36 muestra que es consistente con todos los supuestos de validez y confiabilidad en forma satisfactoria, aunque la traducción de ciertas preguntas se examinará en profundidad para determinar modificaciones subsecuentes.

Palabras clave: estado de salud; encuestas epidemiológicas; México health and vitality. Conclusions. Based on the psychometric evaluation, the SF-36 shows satisfactory consistency with its supposed validity and reliability, although the translation of certain items will be further analyzed for subsequent modifications.

Key words: health status; epidemiologic surveys; Mexico a evaluación de encuestas que integran la percepción del estado de salud de los individuos en actividades de la vida cotidiana ha sido objeto de un creciente interés por parte de la comunidad de investigadores de los servicios de salud. El uso de instrumentos de recolección de datos que miden y caracterizan el estado multidimensional de salud promete el acercamiento en la relación médico-paciente, de forma tal que los pacientes tendrán un nuevo marco de referencia respecto a sus prestadores de servicios de salud, y estos últimos podrán juzgar la efectividad del manejo de la población atendida. ${ }^{1,2}$

La rigurosidad psicométrica de los instrumentos actuales ha permitido la incorporación del concepto de evaluación de la calidad de vida, o lo que otros autores refieren como calidad de vida relacionada con la salud, ${ }^{3}$ y lo ha convertido en la unidad fundamental para la medición de resultados en investigaciones dentro de este campo. Aunque no existe consenso, la calidad de vida relacionada con la salud se mide por medio de la función física, del estado psicológico, de la función y la interacción sociales, y de los síntomas físicos. El espectro de indicadores para la medición de dichos resultados incluye cinco dominios principales: a) mortalidad, b) morbilidad, c) incapacidad, d) incomodidad y e) insatisfacción.

Tradicionalmente, los informes comparativos del estado de salud y enfermedad en diversos grupos poblacionales sólo incluyen datos de mortalidad y morbilidad. En la actualidad, hay una explosión en la literatura médica respecto a la inclusión de las opiniones de los usuarios de servicios médicos para la evaluación de las percepciones del estado de salud. Esta tendencia también se presenta en la literatura mexicana. ${ }^{4-6}$ Las dimensiones de incapacidad, incomodidad e insatisfacción son reconocidas como componentes del concepto de calidad de vida. La incapacidad se mide por la habilidad de realizar actividades y por el impacto físico que esta última produce en la actividad física, en la ocupacional y en la cotidiana. La incomodidad se caracteriza por la presencia o ausencia del dolor físico y la fatiga. La insatisfacción se mide por el funcionamiento social, el bienestar general y la satisfacción con la atención que brindan los prestadores de servicios de salud.

Con el reconocimiento de que, para comparar el estado de salud de las poblaciones de diferentes países se requiere de instrumentos estandarizados, en 1991 se inició el proyecto conocido como "Evaluación internacional de la calidad de vida" (International Quality of Life Assessment Project, IQOLA) para traducir, adaptar y probar la aplicabilidad intercultural de un instrumento genérico denominado Encuesta de Salud SF-36 (Short Form 36 Health Survey)., ${ }^{7.8}$

Inicialmente, este instrumento se usó en el estudio de resultados médicos (Medical Outcomes Study, MOS), donde se demostró su validez y confiabilidad; asimismo, se determinaron las normas de comparación para la población de Estados Unidos de América (EUA). ${ }^{9-12}$

Posteriormente, el proyecto IQOLA incluyó 14 países industrializados, y en la actualidad hay más de 40 naciones participantes. Existen seis versiones de la Encuesta SF-36 en castellano que se han adaptado y utilizado en Argentina, Colombia, España, Honduras y México, así como entre la población méxico-norteamericana de EUA. Los investigadores de estos países tienen como objetivo final la adaptación y el establecimiento de normas de las escalas de la Encuesta SF-36 para su uso en poblaciones genéricas y específicas. La SF-36 se ha utilizado en investigaciones clínicas también específicas; por ejemplo, Alonso y colaboradores informaron sobre la validez y la confiabilidad de una versión en castellano (para usarse en España), a partir de un estudio hecho entre 46 pacientes con cardiopatía coronaria estable. ${ }^{13}$

El presente estudio utilizó la versión 1.1 de la Encuesta, autorizada para usarse en México (SF-36 de aquí 
en adelante), con el fin de medir el estado funcional de una muestra de individuos del sureste del país (véase el anexo).

\section{Material y métodos}

\section{Generalidades de la Encuesta SF-36}

La SF-36 evalúa aspectos de la calidad de vida en poblaciones adultas (mayores de 16 años). El producto de su aplicación es la construcción de ocho conceptos o escalas de salud resultado del promedio de la suma de las preguntas contenidas en el cuestionario. Estos conceptos son: a) función física (FF), b) rol físico (RF), c) dolor corporal (DC), d) salud general (SG), e) vitalidad (VT), f) función social (FS), g) rol emocional (RE) y h) salud mental (SM).

Además de los ocho conceptos de salud, la SF-36 incluye el concepto general de cambios en la percepción del estado de salud actual y en la del año anterior. La respuesta a esta pregunta describe la transición de la percepción respecto al mejoramiento o empeoramiento del estado de salud. En el cuadro I se presenta una descripción de las escalas de salud y sus respecti- vas interpretaciones de acuerdo con resultados bajos o altos por cada escala.

La versión autorizada de la SF-36 para su uso en México siguió un procedimiento estandarizado de traducción/retraducción por expertos bilingües; asimismo, se hicieron análisis por grupos focales representativos y se llevó a cabo una evaluación formal de la calidad de cada traducción. Esta metodología se describe con detalle en otras publicaciones. ${ }^{7,8}$

La SF-36 es un instrumento autoaplicado, contiene 36 preguntas y toma un promedio de 8 a 12 minutos para completarse. Por cada escala, las respuestas a cada pregunta se codifican y recodifican (10 preguntas), y los resultados se trasladan a una escala de 0 (peor salud) a 100 (mejor salud).

\section{Características de la población estudiada}

La SF-36 se aplicó a 257 personas. Del total de encuestados, 87 fueron empleados usuarios de los servicios médicos institucionales de un centro de educación superior ubicado en el sureste de México. Los participantes se seleccionaron aleatoriamente de las listas de pacientes atendidos en los servicios médicos durante

\section{Cuadro I}

Escalas del estado de SALUd e InTERPRetación de Resultados bajos y altos. Encuesta de Salud SF-36

\begin{tabular}{|c|c|c|c|}
\hline \multirow[b]{2}{*}{ Conceptos } & \multirow[b]{2}{*}{ No. de preguntas } & \multicolumn{2}{|c|}{ Significado de los resultados } \\
\hline & & Bajo & Alto \\
\hline Función física & 10 & $\begin{array}{l}\text { Mucha limitación para realizar todas las activida- } \\
\text { des físicas incluyendo bañarse } 0 \text { vestirse debido } \\
\text { a la salud }\end{array}$ & $\begin{array}{l}\text { Realiza todo tipo de actividades físicas, incluyen- } \\
\text { do las más vigorosas, sin limitantes debido a la } \\
\text { salud }\end{array}$ \\
\hline Rol físico & 4 & $\begin{array}{l}\text { Problemas con el trabajo u otras actividades dia- } \\
\text { rias como resultado de la salud física }\end{array}$ & $\begin{array}{l}N \text { ingún problema con el trabajo u otras activida- } \\
\text { des diarias como resultado de la salud física }\end{array}$ \\
\hline Dolor corporal & 2 & Dolor muy severo y extremadamente limitante & Ausencia de dolor o limitaciones debido a dolor \\
\hline Salud general & 5 & $\begin{array}{l}\text { Evalúa la salud personal como mala y cree que } \\
\text { probablemente empeorará }\end{array}$ & Evalúa la salud personal como excelente \\
\hline Vitalidad & 4 & Cansancio y agotamiento to do el tiempo & Lleno de entusiasmo y energía to do el tiempo \\
\hline Función social & 2 & $\begin{array}{l}\text { Interferencia frecuente y extrema con las activi- } \\
\text { dades sociales normales debido a problemas físi- } \\
\text { cos y emocionales }\end{array}$ & $\begin{array}{l}\text { Realiza actividades sociales normales sin interfe- } \\
\text { rencia debido a problemas físicos o emocionales }\end{array}$ \\
\hline Rol emocional & 3 & $\begin{array}{l}\text { Problemas con el trabajo u otras actividades dia- } \\
\text { rias como resultado de problemas emocionales }\end{array}$ & $\begin{array}{l}N \text { ingún problema con el trabajo u otras activida- } \\
\text { des diarias como resultado de problemas emo- } \\
\text { cionales }\end{array}$ \\
\hline Salud mental & 5 & $\begin{array}{l}\text { Sensación de nerviosismo y depresión todo el } \\
\text { tiempo }\end{array}$ & $\begin{array}{l}\text { Sensación de paz, felicidad y calma todo el tiem- } \\
\text { po }\end{array}$ \\
\hline Transición de salud notificada & 1 & $\begin{array}{l}\text { Cree que su salud es mucho mejor ahora que } \\
\text { hace un año }\end{array}$ & $\begin{array}{l}\text { Cree que su salud es mucho peor ahora que hace } \\
\text { un año }\end{array}$ \\
\hline
\end{tabular}


las últimas dos semanas previas a la administración de la encuesta. Se determinó el lugar de trabajo de los seleccionados y se les visitó con el fin de solicitar su participación voluntaria para la autoadministración de la SF-36. Para comparar los resultados de la población blanco, se encuestaron 170 adultos voluntarios que trabajaban en una institución pública, mismos que se seleccionaron aleatoriamente de una lista de 3500 empleados. Los criterios de selección de la población control fueron: a) pertenecer a grupos de edad y sexo similares a la población usuaria de servicios médicos; b) no haber utilizado servicios médicos en los 12 meses previos a la administración de la SF-36, y c) que informaran no padecer de enfermedades crónicas.

\section{A nálisis psicométrico de la Encuesta de Salud SF-36}

En este estudio, la traducción autorizada de la SF-36 para su uso en México se analizó psicométricamente para determinar la validez y la confiabilidad de las escalas.

La validez se define como el grado en el cual el instrumento mide lo que se quiere medir. Cuando se usan instrumentos que producen escalas por la suma de sus componentes (escalas tipo Lykert), como es el caso de la Encuesta de Salud SF-36, se utilizan varios supuestos para medir validez. Entre ellos se encontraron los siguientes: a) una pregunta debe estar linealmente relacionada en forma sustancial con el concepto subyacente medido (validez convergente); $b$ ) una pregunta debe correlacionarse en forma sustancialmente alta con su escala hipotetizada, en lugar de que lo haga con escalas que miden otros conceptos (validez discriminativa); c) preguntas que miden el mismo concepto deben tener aproximadamente igualdad de varianza, para evitar estandarización (prueba de homoscedaticidad), y d) preguntas de una misma escala deben contener similar promedio de información acerca del concepto a medirse (prueba de igual correlación de pregunta-escala). ${ }^{14}$

Las pruebas de confiabilidad y correlación interescalar se usan para determinar si los resultados de las escalas son reproducibles e interpretables. La confiabilidad de un instrumento se refiere al grado en el cual éste se encuentra libre de error aleatorio de medición; sin embargo, este último nunca se elimina, aunque se deben hacer esfuerzos para reducirlo a un nivel mínimo. Se considera que una encuesta es confiable cuando los resultados observados son altamente correlacionados con los resultados verdaderos o hipotetizados. En este estudio se utilizó la prueba de consistencia interna o coeficiente de Cronbach ${ }^{15}(\alpha$ de Cronbach) para determinar confiablidad. Este coeficiente provee un estimado de confiabilidad basado en todas las posibles correlaciones entre dos grupos de preguntas en una prueba. El cálculo del coeficiente $\alpha$ se fundamenta en la correlación media de cada pregunta en cada una de las escalas, con el total de las mismas, y el número de preguntas que contiene.

Se caracterizó a los participantes según el sexo, los grupos de edad y el grupo blanco o control. La información se procesó en una base de datos computarizada y se manipuló con paquetes estadísticos. Las escalas de salud se obtuvieron utilizando los algoritmos propuestos por Ware y colaboradores. ${ }^{8-12}$ El paquete estadístico Multitrait Analysis Program (MAP-R $)^{16}$ se utilizó para la comprobación psicométrica de la encuesta y para determinar la validez de las medidas estadísticas de la adaptación lingüística.

\section{Resultados}

\section{Caracterización de los pacientes participantes en la Encuesta}

De un total de 257 individuos, $33.8 \%$ fueron usuarios de servicios médicos. Con esto se logró una relación de dos controles por cada usuario. La mitad de los participantes $(50.1 \%)$ pertenecía al sexo masculino, y el grupo de edad con mayor frecuencia fue el de 35-44 años $(40.2 \%)$. El rango de edades fue de 18 a 64 años.

Los motivos que limitaron la aplicación de la encuesta a las personas seleccionadas en los servicios médicos y en el grupo control fueron variados; destaca la ausencia de los individuos en su puesto de trabajo en el momento de la visita, así como el temor a que los datos de la Encuesta fueran revelados a sus supervisores. Dos usuarios de los servicios médicos fueron eliminados porque más de $50 \%$ de sus respuestas estaban incompletas, al igual que cinco participantes del grupo control.

\section{Desempeño de la Encuesta}

La construcción de perfiles de los ocho conceptos o escalas de salud es el resultado promedio de la suma sistemática de las preguntas correspondientes a cada escala. Los resultados de los promedios de las escalas se presentan en el cuadro II.

Los promedios de las escalas de salud FF y VT fueron más altos en los hombres que en las mujeres; por otra parte, los de salud general fueron más bajos en los hombres. Las medias de todas las escalas de sa- 


\section{Cuadro II}

Promedios y desviación estándar de las ocho escalas de la SF-36 en usuarios de servicios médicos Y POBLACIÓN CONTROL, POR SEXo Y GRUPOS DE edAD. MéridA,YuCATÁN, MéXICO, 1995

\begin{tabular}{|c|c|c|c|c|c|c|c|c|c|c|c|c|c|c|c|c|}
\hline \multirow{3}{*}{$\begin{array}{l}\text { Población } \\
\text { Servicios médicos }\end{array}$} & \multicolumn{2}{|c|}{ Función física } & \multicolumn{2}{|c|}{ Rol físico } & \multicolumn{2}{|c|}{ Dolor corporal } & \multicolumn{2}{|c|}{ Salud general } & \multicolumn{2}{|c|}{ Vitalidad } & \multicolumn{2}{|c|}{ Función social } & \multicolumn{2}{|c|}{ Rol emocional } & \multicolumn{2}{|c|}{ Salud mental } \\
\hline & $\bar{X}$ & $\mathrm{DE}$ & $\bar{X}$ & $\mathrm{DE}$ & $\bar{x}$ & $\mathrm{DE}$ & $\bar{X}$ & $\mathrm{DE}$ & $\bar{X}$ & $D E$ & $\bar{X}$ & $\mathrm{DE}$ & $\bar{X}$ & $\mathrm{DE}$ & & $\mathrm{DE}$ \\
\hline & 88.9 & (13.8) & 89.2 & (21.6) & 78.4 & (18.7) & 63.2 & (16.7) & 73.9 & (15.6) & 84.2 & (17.4) & 84.1 & (25.1) & 77.4 & $(17.2)$ \\
\hline Hombres & 91 & $(10.2)$ & 89 & (25.4) & 82 & (15.5) & 61 & $(17.9)$ & 77 & (13.1) & 85 & (17.9) & 84 & (23.9) & 77 & (19.9) \\
\hline Mujeres & 87 & (15.6) & 89 & (18.3) & 76 & $(20.9)$ & 65 & $(16.0)$ & 72 & (16.9) & 83 & (17.1) & 84 & (26.4) & 77 & (14.9) \\
\hline 18-24 años & 90 & (6.1) & 83 & $(10.7)$ & 63 & (15.1) & 56 & $(22.2)$ & 85 & (8.6) & 79 & (13.8) & 78 & $(14.0)$ & 73 & $(8.9)$ \\
\hline 25-34 años & 87 & (19.1) & 91 & $(18.9)$ & 78 & $(17.8)$ & 69 & $(16.0)$ & 76 & (14.9) & 87 & (15.3) & 84 & $(25.7)$ & 83 & (11.1) \\
\hline 35-44 años & 90 & (11.8) & 91 & (18.1) & 77 & (19.7) & 63 & $(14.2)$ & 72 & (15.3) & 84 & (19.1) & 82 & $(29.5)$ & 76 & $(13.2)$ \\
\hline 45-54 años & 89 & (11.1) & 82 & (33) & 87 & (16.1) & 60 & $(18.0)$ & 76 & (12.7) & 84 & (17.6) & 87 & $(20.2)$ & 77 & $(27.3)$ \\
\hline 55-64 años & 93 & $(5.2)$ & 100 & $(5.4)$ & 89 & (21.6) & 40 & $(15.9)$ & 50 & (27.6) & 67 & $(17.9)$ & 100 & (7.9) & 53 & $(22.1)$ \\
\hline ntrol & 94.6 & (8.6) & 94.5 & $(15.4)$ & 88.6 & (15.4) & 72.9 & (15.5) & 81.0 & (11.6) & 86.9 & $(15.6)$ & 83.4 & (25.1) & 79.6 & (13.9) \\
\hline Hombres & 95 & (8.1) & 94 & (15.7) & 87 & $(14.6)$ & 72 & $(16.0)$ & 80 & $(12.9)$ & 87 & $(15.7)$ & 80 & $(28.1)$ & 79 & (13.5) \\
\hline Mujeres & 94 & (9.3) & 95 & (15.1) & 90 & (16.3) & 74 & (14.8) & 82 & (9.7) & 87 & (15.7) & 87 & (20.4) & 80 & (14.4) \\
\hline 18-24 años & 93 & $(11.0)$ & 89 & (15.9) & 90 & $(15.0)$ & 80 & $(10.6)$ & 75 & $(15.6)$ & 91 & (14.4) & 87 & $(24.9)$ & 73 & (19.6) \\
\hline 25-34 años & 96 & (6.9) & 95 & $(14.0)$ & 88 & $(13.3)$ & 74 & (14.9) & 79 & $(10.4)$ & 87 & $(16.0)$ & 76 & $(28.5)$ & 79 & (11.1) \\
\hline 35-44 años & 96 & (5.7) & 95 & $(14.6)$ & 90 & $(14.5)$ & 72 & $(16.0)$ & 81 & (11.8) & 88 & (14.3) & 85 & $(24.6)$ & 80 & $(15.0)$ \\
\hline 45-54 años & 90 & (10.5) & 96 & $(11.0)$ & 88 & (12.9) & 71 & $(16.1)$ & 83 & (11.3) & 84 & (15.9) & 90 & (19.5) & 79 & (13.5) \\
\hline 55-64 años & 89 & (19.9) & 84 & $(35.2)$ & 77 & $(35.5)$ & 72 & $(18.2)$ & 87 & $(12.2)$ & 86 & $(25.4)$ & 81 & $(20.7)$ & 85 & $(15.6)$ \\
\hline
\end{tabular}

DE: desviación estándar

lud en los grupos de edad presentados muestran variabilidad sin tendencias específicas.

La escala de los participantes de servicios médicos estudiada con puntuación más alta es la que representa el rol físico (89.2), y la más baja es salud general (63.2). Los controles mostraron la puntuación más alta en FF (94.6) y la más baja en SG (72.9). Se realizaron comparaciones estadísticas (análisis de varianza) de promedios de cada escala de salud entre los participantes de servicios médicos y la población control de las ocho escalas de salud. Esta comparación de promedios mostró que las escalas de salud FF, RF, DC, SG y VT son estadísticamente diferentes en las poblaciones estudiadas. En el cuadro III se indican los resultados de la comparación estadística de escalas de salud en la población participante.

Las escalas de FF, RF, DC, FS y RE representan el estado de salud como la ausencia de limitaciones o incapacidad. El resultado máximo de 100 se logra cuando los participantes no notifican incapacidad alguna. Estas escalas de salud se manifiestan por la esperada unipolaridad de respuestas en el extremo superior del recorrido de 0 a 100 puntos.

Las escalas de SG, VT y SM son bipolares, ya que representan un espectro más amplio de estados de sa-

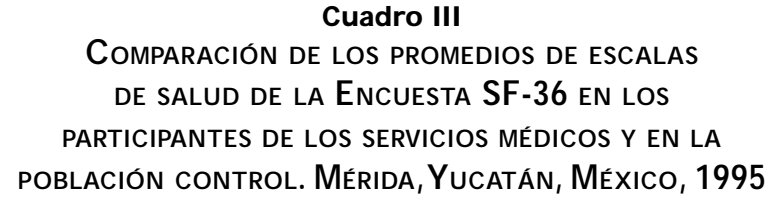

Servicios $\quad \Delta^{*}$ de Grupo $\quad \mathrm{F} \quad$ Probabilidad médicos control y SM

\begin{tabular}{lrrrr} 
Función física & 88.9 & +5.7 & 12.4 & 0.000 \\
\hline Rol físico & 89.3 & +5.2 & 4.8 & 0.029 \\
\hline Dolor corporal & 78.4 & +10.2 & 21.3 & 0.000 \\
\hline Salud general & 63.2 & +9.8 & 16.2 & 0.000 \\
\hline Vitalidad & 73.9 & +7.2 & 15.0 & 0.000 \\
\hline Función social & 84.2 & +2.7 & 1.6 & 0.210 \\
\hline Rol emocional & 84.1 & -0.7 & 0.1 & 0.829 \\
\hline Salud mental & 77.4 & +2.2 & 1.2 & 0.270
\end{tabular}

SM: servicios médicos

* Diferencia entre promedio del grupo control y el grupo de servicios médicos

lud positivos y negativos. Estas escalas requieren de un informe favorable a partir de la evaluación del estado de salud para alcanzar el resultado más alto posi- 
ble. Las respuestas encontradas para estas escalas se concentran alrededor del segundo cuartil, y muy pocas respuestas están comprendidas en los extremos. Los individuos que completaron la SF-36 utilizaron el espectro total de respuestas en 19 de las 36 preguntas. Las respuestas que representan los más bajos niveles de salud no se manifestaron en muchas de las preguntas. Las escalas de rol físico y salud general se usan como ejemplo en la figura 1, para mostrar la unipolaridad y la bipolaridad de las respuestas.

El concepto general de cambios en la percepción del estado de salud actual y la del año anterior mostró respuestas similares en ambos grupos estudiados. El mayor porcentaje de las respuestas estuvo entre las opciones "algo mejor ahora que hace un año" y "más o menos igual que hace un año".

\section{Validez y confiabilidad de la Encuesta de Salud SF-36}

La validez convergente de las preguntas fue, en general, comprobada con una correlación de $0.40^{14}$ o mayor con su escala hipotetizada. En seis preguntas se encontraron problemas, mismos que se describen a continua-
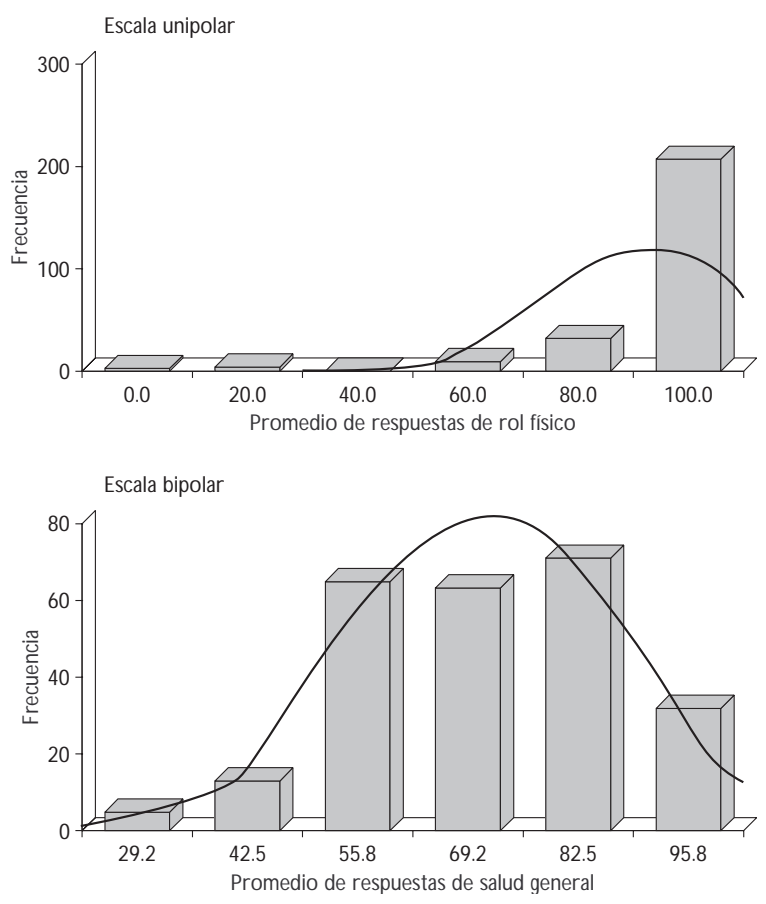

Figura 1. Distribución de respuestas de la escala UNIPOLAR (ROL FÍSICO) Y LA ESCALA BIPOLAR (SALUD GEneral). Mérida, Yucatán, México, 1995 ción. En tres preguntas se observaron dificultades de medición y en otra se encontró que todas las respuestas fueron similares, lo que invalida el análisis de contribución de respuestas a la construcción de la escala de salud. En una pregunta más se encontró que no existió correlación con el concepto hipotetizado, lo que se consideró como un posible problema de traducción o interpretación por circunstancias culturales, y en una sexta pregunta hubo baja correlación con las otras preguntas que se usan para construir la escala. La evaluación de la homoscedasticidad muestra que 35 de las 36 preguntas en cada escala contribuyen de manera similar a sus respectivas escalas. Las otras suposiciones de validez mostraron, en general, resultados excelentes.

Las pruebas estadísticas de confiabilidad medidas por la consistencia interna del cuestionario muestran un Coeficiente $\alpha$ de Cronbach con un rango de 0.56 a 0.84 (cuadro IV). Los coeficientes estuvieron entre $0.70^{14}$ (valor mínimo recomendado para efectos comparativos) para todas las escalas excepto para rol emocional, el cual mostró una correlación de 0.56. La mayoría de las correlaciones interescalares resultaron en rangos intermedios. Las correlaciones más altas se observaron en VT, FS, SM (rango 0.54-0.73). De las cuatro escalas de salud representativas del constructo salud mental, el rol emocional tuvo las correlaciones más bajas. Las correlaciones entre la función física y las demás escalas también fueron bajas.

\section{Discusión}

Las encuestas que enfocan la medición de diferentes aspectos del estado de salud deben ser cortas, aplicables a diversos grupos poblacionales, preferiblemente autoadministradas, y sus resultados deben presentar

\section{Cuadro IV \\ Consistencia interna ( $\alpha$ de Cronbach) $y$ correlación interescalar de la Encuesta de Salud SF-36. Mérida, Yucatán, México, 1995}

$\alpha$ de Cronbach FF RF DC SG VT FS RE

\begin{tabular}{llllllllll} 
Función física & .84 & & & & & & & \\
\hline Rol físico & .70 & .43 & & & & & & \\
\hline Dolor corporal & .80 & .42 & .37 & & & & & \\
\hline Salud general & .73 & .18 & .33 & .40 & & & & \\
\hline Vitalidad & .83 & .26 & .31 & .45 & .47 & & & \\
\hline Función social & .76 & .15 & .48 & .48 & .40 & .65 & & \\
\hline Rol emocional & .56 & .04 & .33 & .25 & .16 & .31 & .54 & \\
\hline Salud mental & .83 & .09 & .24 & .27 & .41 & .73 & .69 & .33
\end{tabular}


de manera clara los aspectos de salud medidos. La SF36 tiene el potencial de cumplir con estos requerimientos; además, permite la evaluación multidimensional del concepto de salud e incluye aspectos de incapacidad, incomodidad e insatisfacción, componentes todos de la evaluación de la calidad de vida relacionada con la salud.

Los hallazgos de esta encuesta demuestran, en promedio, la percepción de buena salud de los participantes. En este estudio no se observó la tendencia a promedios más bajos en las escalas de salud al incrementar la edad de los participantes. El alto promedio de respuestas comprendidas en el techo de las escalas FF, RF, FS y RE reflejan la observación de "buena sa-

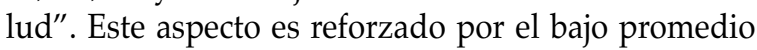
de respuestas en los extremos de las tres escalas bipolares (SG, VT, y SM) en las cuales se miden incapacidad y bienestar en general. Este hecho también contribuye a la conclusión de que los participantes procedentes de los servicios médicos representan a un grupo de población relativamente saludable. Los resultados que se presentan aquí muestran que las escalas FF, RF, DC, SG y VT son estadísticamente más altas en la población control que en la que asistió a los servicios médicos. Estas diferencias son la base para establecer normas para grupos de población específicos.

El uso potencial de la SF-36 para comparar poblaciones en diferentes áreas geográficas, en distintos periodos cronológicos y en diferentes sistemas de servicios de salud, es de extraordinario valor. Con base en los resultados de la evaluación psicométrica, queda demostrado que la Encuesta es consistente con todos los supuestos de validez y confiabilidad en forma satisfactoria, si bien la traducción de ciertas preguntas se examinará a profundidad para determinar modificaciones en mediciones subsecuentes. La escala de salud que mide el RE presenta las resultantes que son más difíciles de abordar en este estudio. El promedio de RE para los participantes de los servicios médicos fue 0.7 puntos promedio mayor que para el grupo control, lo que la convirtió en la única escala con mayor promedio en el grupo que hipotéticamente representa a una población menos saludable. Además, la consistencia interna de la escala RE es la única que está por debajo del nivel mínimo aceptable para comparaciones de grupo. Entre las posibles explicaciones para estos hallazgos se encuentra la eventualidad de que sea: a) un hallazgo casual; b) un problema en la traducción; c) el hecho de que la población haya sido muy homogénea en términos de "buena salud", y / o d) la existencia de aspectos culturales que modulan la forma como se interpretan las preguntas que conforman esta escala.
En este estudio no se encontró evidencia para modificar los algoritmos usados en la construcción de las escalas de salud, por lo que se recomienda la utilización de los mismos. En todos los casos se autoaplicó la SF-36. Los estudios en los que se busque evaluar su aplicación mediante un entrevistador, están garantizados en poblaciones específicas que requerirán de métodos alternos de administración debido a factores tales como distancia, analfabetismo, dificultad de visión y / $\mathrm{u}$ otras incapacidades físicas o funcionales. En estudios realizados por el MOS se ha comprobado que la intervención de entrevistadores para administrar la encuesta SF-36 da como resultado mejores perfiles de salud en todas las escalas, ${ }^{17}$ lo anterior considerando el sesgo introducido por el entrevistador.

\section{Agradecimientos}

Se agradecen los acertados comentarios de los revisores anónimos.

\section{Referencias}

1. Patrick DL, Bergner M. Measurement of health status in the 1990 's.Ann Rev Public Health 1990;11:165-183.

2.W are JE Jr.The status of health assessment 1994.Ann Rev Public Health 1995;16:327-354.

3. G uyatt GH, Feeney DH, Patrick DL. Measuring health related to quality of life. Ann Intern Med 1993;118:622-629.

4.Vandale-Toney S, D urán-Arenas JLG , 0 rtega-Minor H. La medición del estado de salud de la población: una actividad fundamental para los servicios de salud. Salud Publica Mex 1985;27(2):116-123.

5. Schlaepfer-Pedrazzini L, Infante-C astañeda C. La medición de la salud: perspectivas teóricas y metodológicas. Salud Publica Mex 1990;32(2): 141-155.

6. Ruiz de Chávez M, Martínez-N arvaez G, Manuel C alvo-Ríos J,A guirre$G$ as $H$, A rango-Rojas R, Lara- $C$ arreno $R$, et al. Bases para la evaluación de la atención de las unidades médicas del sector salud. Salud Publica Mex 1990;32(2):156-169.

7.Aaronson N K,A cquadro C,Alonso J,A polone G, Eucquet D, Bullinger M, et al. International quality of life assessment (IQ $O \mathrm{LA}$ ) project. Q ual Life Res 1992;1:349-351.

8. W are J, Keller S, Gandek B, Brazier J, Sullivan M, the IQ O LA Project $G$ roup. Evaluating translation of health status questionnaires: Methods from the IQ O LA Project. Int J Technol Assess Health C are 1995;11(3):525-551. 9.W are JE. SF-36 health survey manual and interpretation guide. Boston: The Health Institute, N ew England Medical Center, 1993.

10.W are JE, Sherbourne CD.The MOS 36-item short-form health survey (SF-36). I. Conceptual framework and item selection. Med Care 1992;30: 473-483.

11. MCHorney CA, W are JE, Raczek AE. The MOS 36-item short-form health survey (SF-36). II. Psychometric and clinical tests of validity in measuring physical and mental health constructs. Med C are 1993;31:247-263. 12. McH orney CA, Kosinski M, W are JE. The MOS 36-item short-form health survey (SF-36). III. Tests of data quality, scaling assumptions, and reliability across diverse patient groups. Med Care 1994;32(1):551-567. 
13. Alonso J, Prieto L, Anto JM. The Spanish version of the SF-36 health survey: An instrument for measuring clinical results. Med Clin 1995; 104(20):771-776.

14. Campbell DT, Fiske DW. Convergent and discriminant validation by the multitrait-multimethods matrix. Psychol Bull 1959;56:81-105.

15. Cronbach $L$ J. C oefficient alpha and the internal structure of tests. Psychometrika 1951;16:297-334.
16. Hays RD, Hayashi T, C arson S,W are JE. User's guide for the multitrait analysis program (MAP), RAND N ote N-2786-RC. Santa Mónica (CA): RAN D Corporation, 1968.

17. MCHorney CA, Kosinski $M, W$ are JE. Comparisons of the cost and quality of norms for the SF-36 health survey collected by mail versus telephone interview: Results from a national survey. Med Care 1994;32(6): 551-567. 


\section{Anexo \\ Encuesta del Estado de Salud SF-36}

La Encuesta del Estado de Salud SF-36 para su uso en México se puede reproducir con permiso del Health Assessment Lab, $\mathrm{N}$ ew England Medical Center, 750 W ashington Street, N EMC \#345, Boston, MA, 02111. D erechos Reservados @ Health Assessment Lab. El permiso para el uso de la Encuesta puede ser solicitado al primer autor de este artículo.

Instrucciones: esta encuesta le pide su opinión acerca de su salud. Esta información permitirá saber cómo se siente y qué tan bien puede hacer usted sus actividades normales.

Conteste cada pregunta marcando la respuesta como se le indica. Si no está seguro o segura de cómo responder a una pregunta, por favor dé la mejor respuesta posible.

1. En general, ¿diría que su salud es:

Escalas de respuesta: Excelente, Muy buena, Buena, Regular, Mala?

2. Comparando su salud con la de hace un año, ¿cómo la calificaría en general ahora?

Escalas de respuesta: Mucho mejor ahora que hace un año. Algo mejor ahora que hace un año. Más o menos igual ahora que hace un año.Algo peor ahora que hace un año. Mucho peor ahora que hace un año.

3. Las siguientes frases se refieren a actividades que usted podría hacer durante un día normal. ¿Su estado de salud actual lo limita para hacer estas actividades? Si es así, ¿cuánto?

Escalas de respuesta: Sí, me limita mucho. Sí, me limita un poco. N o, no me limita en absoluto.

0 pciones de pregunta:

a. Actividades vigorosas, tales como correr, levantar objetos pesados, participar en deportes intensos.

b. Actividades moderadas, tales como mover una mesa, barrer, trapear, lavar, jugar futbol 0 beisbol.

c. Levantar o llevar las compras del mercado.

d. Subir varios pisos por la escalera.

e. Subir un piso por la escalera.

f. Doblarse, arrodilarse 0 agacharse.

g. Caminar más de diez cuadras.

h. Caminar varias cuadras.

i. Caminar una cuadra.

j. Bañarse 0 vestirse.

4. Durante el último mes, iha tenido usted alguno de los siguientes problemas con el trabajo u otras actividades diarias normales a causa de su salud física?

Escalas de respuesta: Sí, No

0 pciones de pregunta:

a. Ha reducido el tiempo que dedicaba al trabajo u otras actividades.

b. Ha logrado hacer menos de lo que le hubiera gustado.

c. Ha tenido limitaciones en cuanto al tipo de trabajo u otras actividades.

d. Ha tenido dificultades en realizar el trabajo u otras actividades (por ejemplo, ha requerido de mayor esfuerzo).

5. Durante el último mes, iha tenido usted alguno de los siguientes problemas con el trabajo u otras actividades diarias normales a causa de algún problema emocional (como sentirse deprimido 0 ansioso?

Escalas de respuesta: Sí, N o
0 pciones de pregunta:

a. Ha reducido el tiempo que dedicaba al trabajo u otras actividades.

b. Ha logrado hacer menos de lo que le hubiera gustado.

c. Ha hecho el trabajo u otras actividades con el cuidado de siempre.

6. Durante el último mes, ¿en qué medida su salud física o sus problemas emocionales han dificultado sus actividades sociales normales con la familia, amigos, vecinos o grupos?

Escalas de respuesta: $\mathrm{N}$ ada. Un poco. Más o menos. Mucho. Demasiado.

7. ¿Cuanto dolor físico ha tenido usted durante el último mes? Escalas de respuesta: $N$ ingún dolor. Muy poco. Poco. Moderado. Severo. Muy severo.

8. Durante el último mes, icuánto el dolor le ha dificultado su trabajo normal (incluyendo tanto el trabajo fuera de casa como los quehaceres domésticos)?

Escalas de respuesta: $\mathrm{N}$ ada. Un poco. Más o menos. Mucho. Demasiado.

9. Estas preguntas se refieren a cómo se ha sentido usted durante el último mes. Por cada pregunta, por favor dé la respuesta que más se acerca a la manera como se ha sentido usted. ¿C uánto tiempo durante el último mes

Escalas de respuesta: Siempre. C asi siempre. Muchas veces. Algunas veces. C asi nunca. $\mathrm{N}$ unca.

0 pciones de pregunta:

a. Se ha sentido lleno de vida?

b. Se ha sentido muy nervioso?

c. Se ha sentido tan decaído que nada podía alentarlo?

d. Se ha sentido tranquilo y sosegado?

e. Ha tenido mucha energía?

f. Se ha sentido desanimado y triste?

g. Se ha sentido agotado?

h. Se ha sentido feliz?

i. Se ha sentido cansado?

10. D urante el último mes, ¿cuánto tiempo su salud física o sus problemas emocionales han dificultado sus actividades sociales (como visitar amigos, parientes, etc.)?

Escalas de respuesta: Siempre. Casi siempre. Algunas veces. Casi nunca. N unca.

11. ¿Q ué tan CIERTA o FALSA es cada una de las siguientes frases para usted?

Escalas de respuesta: Definitivamente cierta. Cierta. No sé. Falsa. D efinitivamente falsa.

O pciones de pregunta:

a. Parece que yo me enfermo un poco más fácilmente que otra gente.

b. Tengo tan buena salud como cualquiera que conozco.

c. Creo que mi salud va a empeorar.

d. Mi salud es excelente. 